\title{
The effects on pain, physical function, and quality of life of quadriceps strengthening exercises combined with Baduanjin qigong in older adults with knee osteoarthritis: a quasi-experimental study
}

Fenglan Wang ${ }^{1,2} \mathbb{1}$, Xiaoli Zhang ${ }^{2}$, Xiao Tong ${ }^{3}$, Min Zhang ${ }^{2}$, Fengmei Xing ${ }^{2}$, Kun Yang ${ }^{2}$, Nana Jiao ${ }^{2}$ and Zhiguang Duan ${ }^{1 *}$

\begin{abstract}
Background: Exercise is recommended as a principal treatment for individuals with knee osteoarthritis (KOA). However, the best choice for an optimal exercise program able to promote long-term compliance in KOA patients is not clear. This study aims to compare the effect of combined exercise (CE: quadriceps strengthening exercises (QSE) and Baduanjin qigong training (BDJ)) versus QSE alone and BDJ alone on older adults with KOA.

Methods: A three-arm, quasi-experimental trial with repeated measurements was used. As a cluster randomized trial, participants from three community centers were assigned respectively to QSE group, BDJ group and CE group. We assessed pain intensity, physical function, self-efficacy, and health-related quality-of-life (HRQoL) using standardized instruments at baseline, 3 months and 6 months follow-up.

Results: One hundred and twenty-eight participants with KOA aged over 60 completed the study. Over the 6 months, there were significant group interaction effects on pain intensity $(F=28.888, P<0.001)$, physical function $(F=26.646, P<0.001)$, and self-efficacy $(F=22.359, P<0.001)$, and, based on a short form-12 item health survey questionnaire (SF-12), physical component summary $(F=7.470, P<0.001)$, and mental component summary $(F=$ $10.207, P<0.001)$. Overall, the CE group exhibited significantly greater improvement in all outcomes when compared to the QSE group and the BDJ group.
\end{abstract}

Conclusions: CE treatment is more effective than QSE and BDJ in pain relief, increasing physical function, improving self-efficacy, and raising quality-of-life in community-dwelling KOA older adults. Moreover, it promotes long-term compliance in KOA community patients.

Trial registration: Chinese Clinical Trails Registry number ChiCTR2000033387 (retrospectively registered). Registered 30 May 2020.

Keywords: Knee osteoarthritis, Baduanjin qigong, Quadriceps strengthening exercises

* Correspondence: dzg528@sxmu.edu.cn

'School of Nursing, Shanxi Medical University, 56 Xinjian Road, Yingze District, Taiyuan 030001, China

Full list of author information is available at the end of the article

C C The Author(s). 2021 Open Access This article is licensed under a Creative Commons Attribution 4.0 International License, which permits use, sharing, adaptation, distribution and reproduction in any medium or format, as long as you give appropriate credit to the original author(s) and the source, provide a link to the Creative Commons licence, and indicate if changes were made. The images or other third party material in this article are included in the article's Creative Commons licence, unless indicated otherwise in a credit line to the material. If material is not included in the article's Creative Commons licence and your intended use is not permitted by statutory regulation or exceeds the permitted use, you will need to obtain permission directly from the copyright holder. To view a copy of this licence, visit http://creativecommons.org/licenses/by/4.0/. The Creative Commons Public Domain Dedication waiver (http://creativecommons.org/publicdomain/zero/1.0/) applies to the data made available in this article, unless otherwise stated in a credit line to the data. 


\section{Background}

Osteoarthritis (OA), the third highest risk factor for disability in the elderly, can lead to pain, loss of function, and reduced quality-of-life (QoL) [1]. As the global population ages, the number of elderly with $\mathrm{OA}$ is increasing in both low- and high-income countries [2]. Globally, knee osteoarthritis (KOA) accounts for over $80 \%$ of OA patients [3]. In the USA, KOA affects approximately $37 \%$ of American adults aged over 60 years [4]. In China, years lived with disability (YLDs) for KOA per 100,000 population was 968 in 2012, with $60 \%$ of YLDs contributed by individuals aged over 60 years [5].

As there is no way to cure KOA [1], treatment of KOA mainly focuses on pain reduction and improving physical function and quality-of-life [6, 7]. Exercise therapy is perhaps the most efficient non-pharmacologic treatment for KOA due to its low-cost, high safety, and facile operation [7, 8]. The KOA clinical guidelines issued by the American College of Rheumatology, states exercise interventions, including aerobic, resistance, and aquatic exercise are highly recommended [9].

Among recommended exercise programs, quadriceps strengthening exercise (QSE) has been used widely in KOA patients. Previous studies indicate QSE has better short-term effects on the relief of joint pain and stiffness and in improving QoL $[7,10,11]$. However, long-term adherence to exercise regimens is a barrier to the widespread deployment of QSE $[11,12]$. For example, $44.2 \%$ of KOA patients withdrew from exercise intervention due to time, complexity of the intervention program, or other reasons [10]. The type of exercise preferred by patients and the way in which exercise is delivered may affect adherence [12]. Thus, the optimal way to promote long-term exercise adherence in KOA patients must be identified.

Mind-body exercises including Tai Chi, Yoga, Baduanjin, and Qigong have become increasing popular in pain management [13]. When managing KOA, Tai Chi is recommended as an appropriate exercise intervention [9]. Baduanjin, a well-known form of traditional Chinese Qigong, is similar to Tai Chi but requires fewer movements and a shorter time [14]. Studies indicate that Baduanjin has physiological and psychological effects in various disease states, reducing the symptoms of morning stiffness, spinal pain, and fatigue for patients with ankylosing spondylitis [15], alleviated musculoskeletal pain in older people with chronic low back and neck pain $[16,17]$, and improving mental health in many participants [18]. A recent review assessing the safety and efficacy of Baduanjin for KOA found only three randomized controlled trials [14]. It showed that Baduanjin can be a simple and appropriate low-intensity aerobic exercise for elderly KOA patients, but the effect of Baduanjin on improving OA symptoms requires additional investigation.
A review indicates that traditional exercise training focuses on improving muscles strength rather than modulating balance deficits and stress management, which are key factors related to the mobility of KOA patients [19]. Baduanjin, as a mind-body exercise modality, when combined with QSE, may enhance muscles strength, balance, mental health, and stress management.

The Ottawa panel clinical practice guidelines for managing KOA point out that the combination of aerobic exercise and strengthening exercises can significantly improve pain relief and physical function [20]. However, relatively little robust research has been undertaken on the combination of Baduanjin with QSE in KOA patients. This study aims to explore the effect of combining Baduanjin with QSE on older adults with KOA. Our hypothesis is that a combination exercise program could relieve pain and improve physical function, consequently improving self-efficacy and QoL, while also promoting long-term compliance to the exercise regime. Should the outcome of testing this hypothesis be valid, our study would suggest to clinical staff and rehabilitation therapists that when they write exercise prescriptions for KOA patients, rather than focusing solely on exercise programs to improve muscle strength, they should pay equal attention to combined exercise programs producing synergistic effects.

\section{Methods \\ Study design}

This was a quasi-experimental, assessor-blinded trial comprising three parallel groups with repeated measurements. The study was conducted in three community health centers in Tangshan city, China, labelled community $\mathrm{A}$, community $\mathrm{B}$, and community $\mathrm{C}$. Post-test data were collected at 3 months and 6 months after baseline. Prior to any interventions, all researchers received professional training to provide QSE and Baduanjin instruction to the subjects.

\section{Participants}

This study recruited community-dwelling elderly patients with KOA using print and social-media advertisements. After being registered in community centers by research assistants, clinicians screened volunteers using defined inclusion and exclusion criteria to determine initial study participants.

Inclusion criteria were: participants were (1) aged over 60 years, (2) clinically diagnosed with KOA, (3) had experienced knee pain on most days of the previous month, and (4) knee pain in the past week was between 3 and 7 on an 11-point numeric rating scale (NRS). Exclusion criteria were: (1) severe/uncontrolled comorbidities, such as myocardial ischemia, unstable angina pectoris, or mental illness, (2) neurological disorders 
affecting the lower limbs, (3) joint replacement surgery, (4) acute trauma of knee joint, (5) severely deformed lower limbs, and (6) having received intraarticular injection within the past 3 months. As participants should have received no Baduanjin qigong training or quadriceps muscle strength exercise before entering the trial, if subjects performed any kind of sports for at least $20 \mathrm{~min}$ twice a week, for more than 1 month regularly, they were categorized as regular exercisers, and were also excluded.

\section{Randomization and blinding}

To avoid contamination effects among participants from the same community, we used randomization by community, randomizing the three communities by drawing lots. Nine equal-sized sheets of paper read either "QSE intervention in community $\mathrm{A}$ " or "QSE intervention in community B" or "QSE intervention in community C" or "BDJ intervention in community A" or "BDJ intervention in community B" or "BDJ intervention in community C" or " $\mathrm{CE}$ intervention in community $\mathrm{A}$ " or " $\mathrm{CE}$ intervention in community B" or "CE intervention in community $\mathrm{C}^{\prime}$. These sheets were packaged into groups of three and placed into three opaque envelopes. After recruitment, two envelopes were selected randomly and opened by a research assistant. Assessors and statisticians were blinded to participant allocation. They did not participate in the recruitment or intervention process.

\section{Sample size}

The required sample size was calculated using Power Analysis and Sample Size (PASS 15) software set for repeated measures. The primary outcome was defined as change in the pain dimension of the Western Ontario and McMaster Universities Arthritis Index (WOMAC) among the three study groups at the end of three and 6 months. Assuming that the mean difference and standard deviation (SD) among the study groups was equivalent to that from a prior study on community-dwelling KOA patients [21], a minimum of 31 participants per group were required for a medium effect size at a power of $80 \%$ and a significance level of 0.05 . To account for a projected dropout rate of $15 \%$, we sought to include 50 participants per group.

\section{Interventions}

Combined exercise (CE: quadriceps strengthening exercises and Baduanjin qigong) The CE group comprising participants who undertook QSE plus Baduanjin training program at least three times weekly over 6 months. They were asked to complete a brief exercise diary, which recorded the number of exercises per day. The combined QSE and Baduanjin training consisted of two phases: a face-to-face phase (0-6 weeks) and a follow-up phase (6 months). In the face-to-face phase, participants attended a $2 \mathrm{~h}$ class conducted by trained researchers twice weekly for 6 weeks. The main points of QSE and Baduanjin were explained and step-by-step instruction provided. Each class was conducted in groups of 10-15 participants. Each class included an hour for QSE and an hour for Baduanjin. Outside of these sessions, participants were encouraged to practice the exercises themselves. In the follow-up phase, participants were expected to practice QSE at home and practice Baduanjin in groups, at least three times weekly until the end of the study. Scheduled follow-up telephone calls were undertaken each week by research assistants in weeks 7 to 12 , and then each month in weeks 13 to 24 . Telephone calls discussed participant progress, identifying barriers, and encouraged subjects to adhere to the exercise program. Participants were also encouraged to contact researchers should they have concerns or questions.

Quadriceps strengthening exercises (QSE) The QSE program was created after a literature review [22, 23], clinical practice, and consultation with experts. It has been shown to be safe, effective, and straightforward for study participants. QSE exercises undertaken are detailed in Additional file 1. The participants with KOA were asked to practice QSE 30-40 min per day for at least 3 days per week.

Baduanjin qigong All Baduanjin exercises used were recommended by the Chinese Health Qigong Association in 2003, and consisted of eight postures [14]. It was recommended to practice two sets, once per day [14, 15], requiring approximately $20 \mathrm{~min}$ per set. Participants were asked to practice Baduanjin in a group-based form at least 3 days per week, except for those unable to leave home, who practiced at home. Participants were told that exercises should be performed within a tolerable level of pain. If discomfort was experienced while exercising, researchers would undertake a re-assessment, adjusting existing plans based on participants' physical functioning and knee symptoms.

Baduanjin qigong training (BDJ) The BDJ group were trained to use Baduanjin qigong exercising and received telephone follow-ups similar to those of the CE group. During the trial period, participants in the BDJ group did not practice quadriceps strengthening exercises or other types of qigong exercise, Tai Chi, or other similar exercises. However, their daily activities were not otherwise restricted. 
Quadriceps strengthening exercises (QSE) The QSE group were trained to use QSE exercises and had telephone follow-ups similar to those received by the $\mathrm{CE}$ group. During the trial period, participants in the QSE group did not practice Baduanjin or other types of qigong exercise, Tai Chi, or other similar exercises. Their daily activities were not otherwise restricted.

\section{Outcome measurement}

Outcome measures were assessed at baseline, 3 months, and 6 months by the same research assistants who had previously been blinded to intervention allocation. Demographic variables were age, gender, height, weight, body mass index (BMI), disease duration, and social circumstances.

\section{Primary outcome measures}

The primary outcomes were: pain intensity and physical function related to KOA, as measured by WOMAC [24]. The WOMAC index was designed for use in clinical trials of patients with osteoarthritis of the hip and knee. It includes 5 items on pain and 17 items on physical function, rated on a $0-4$ Likert scale. Higher scores reflect greater pain, stiffness, and increased difficulty in physical function. The internal reliability of the Chinese version of WOMAC, as measured using Cronbach's alpha, is $0.67-0.82$ for its three subscales [25].

\section{Secondary outcome measures}

Participants' confidence in managing arthritis symptoms was measured using the 8-item version of the Arthritis Self-Efficacy Scale (ASES-8) [26]. This consists of eight items with no subscales. Responses were averaged, producing a score from 1 (very uncertain) to 10 (very certain), thus a high score indicated greater self-efficacy. The internal consistency of the Chinese ASES-8, as measured by Cronbach's alpha, is 0.942 [27].

HRQoL was measured using a Short Form 12 questionnaire (SF-12), comprising 12 items within two domains: physical component summary (PCS) and mental component summary (MCS) [28]. Higher scores indicate better physical and mental health [29]. SF-12 has been widely used to assess HRQoL in patients with various conditions, including KOA disease, demonstrating good reliability and validity [30]. The Chinese SF-12 has good internal consistency, with a Cronbach's $\alpha$ of 0.86 [29].

\section{Statistical analysis}

Data were analyzed using SPSS version 23.0 (IBM Corporation, Armonk, NY, USA). Descriptive statistics such as means, SDs, frequencies, and percentages, were used to summarize participant demographic and clinical characteristics. For intervention effects, repeated measure analysis of variance was used to determine the significance of differences at 6 months in pain, physical function, self-efficacy, and HRQoL among the three groups. The interaction effect (group $\times$ time) assessed the change among groups in outcome variables across three time points. A Sidak statistical adjustment was used to minimize possible errors from multiple pairwise comparisons. The statistical significance level was set at 0.05 .

\section{Results}

One hundred and seventy-eight eligible participants from three community centers were assessed. Thirty-five were excluded: 19 did not meet selection criteria and 16 did not wish to participate. This left 143 participants who met the study criteria and agreed to participate, with 50 in the CE group, 47 in the QSE group and 46 in the BDJ group. During the study, 15 of the 143 participants were lost to follow-up (10.5\%), as shown in Fig. 1.

\section{Participant characteristics}

Demographic characteristics of the participants are given in Table 1. The mean age was $65.34(\mathrm{SD}=3.17$; range 60-72). $84.4 \%$ of subjects were female and $15.6 \%$ were male. The mean duration of symptoms was 8.02 (SD = 3.73; range 2-16). 32.0\% of participants had two affected knees. There were no statistically significant differences in demographic or clinical data among the three groups at baseline (Table 1). At 6 months, five participants (11.4\%) exercised less than three times per week in the CE group, 13 subjects (30.2\%) in QSE group and nine subjects $(22.0 \%)$ in BDJ group. There were statistically significant differences between CE and QSE groups $\left(\chi^{2}=4.719, P=0.036\right)$. No statistically significant differences were observed between BDJ vs QSE $\left(\chi^{2}=0.745\right.$, $P=0.270)$ and BDJ vs CE groups $\left(\chi^{2}=1.729, P=0.153\right)$.

\section{Effects on WOMAC pain intensity and physical function} No statistically significant differences among the three groups were found for pain intensity or physical function at baseline and at month 3 of follow-up (see Table 2). At month 6 of follow-up, there were statistically significant differences among groups for both pain $(\mathrm{F}=9.071, P<$ $0.001)$ and physical function $(\mathrm{F}=5.440, P=0.005)$.

Repeated measures-ANOVA was undertaken to test for time-by-group-interaction effects on pain intensity and physical function for the length of the whole study. Results are presented in Table 2. This analysis indicated significant time-by-group-interaction effects on pain $(\mathrm{F}=28.888, P<0.001)$ and physical function $(\mathrm{F}=26.646$, $P<0.001)$.

Figures 2 and 3 illustrate time-by-group-interaction effects and show the trends for pain and physical function, across the three study time points. Among the three groups, scores for pain and physical function at the end 


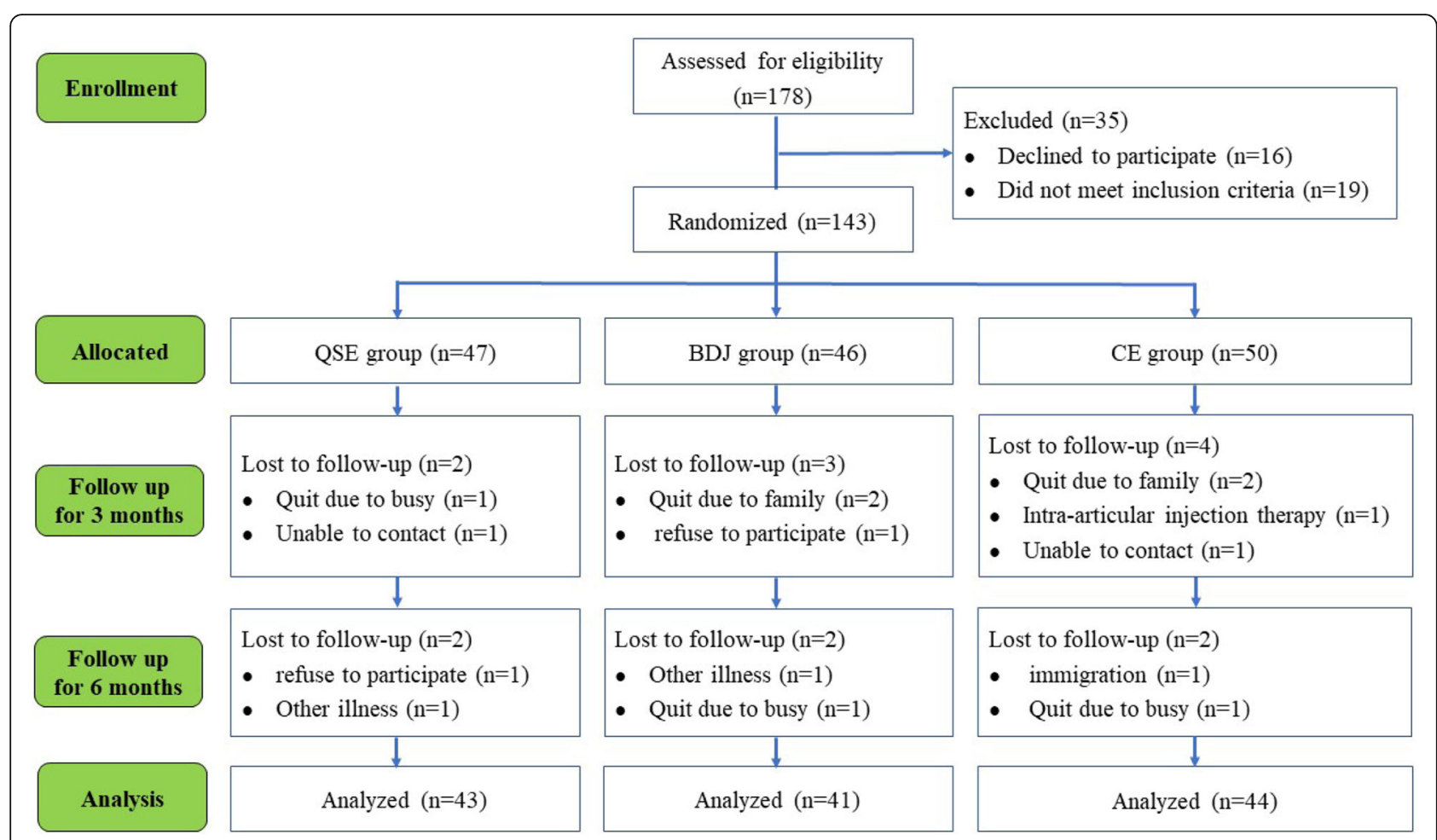

Fig. 1 Flowchart of the study participants. QSE: quadriceps strengthening exercise; BDJ: Baduanjin qigong; CE: the combined exercise of quadriceps strengthening exercise and Baduanjin qigong

Table 1 Baseline demographic and clinical characteristics among the three groups $(n=128)$

\begin{tabular}{|c|c|c|c|c|c|c|}
\hline Characteristic & Total $(n=128)$ & $\mathrm{QSE}(n=43)$ & $\mathrm{BDJ}(n=41)$ & $\mathrm{CE}(n=44)$ & $x^{2 / F}$ & $P$-value \\
\hline Age, Mean (SD) & $65.34 \pm 3.17$ & $65.70 \pm 3.50$ & $64.74 \pm 2.80$ & $65.57 \pm 3.15$ & $1.206^{a}$ & 0.303 \\
\hline Gender $(n, \%)$ & & & & & $0.961^{b}$ & 0.619 \\
\hline Male & $20(15.6)$ & $8(18.6)$ & $7(17.1)$ & $5(11.4)$ & & \\
\hline Female & $108(84.4)$ & $35(81.4)$ & $34(82.9)$ & $39(88.6)$ & & \\
\hline Marital status (n, \%) & & & & & $0.642^{b}$ & 0.725 \\
\hline Married & $109(85.2)$ & $37(86.0)$ & $36(87.8)$ & $36(81.8)$ & & \\
\hline Single/Divorce/ Widowed & $19(14.8)$ & $6(14.0)$ & $5(12.2)$ & $8(18.2)$ & & \\
\hline Educational level $(n, \%)$ & & & & & $0.583^{b}$ & 0.997 \\
\hline Primary education or blow & $27(21.1)$ & $9(20.9)$ & $8(19.5)$ & $10(22.7)$ & & \\
\hline Secondary education & $50(39.1)$ & $18(41.9)$ & 15 (36.6) & $17(38.6)$ & & \\
\hline Higher education & $35(27.3)$ & $11(25.6)$ & $12(29.3)$ & $12(27.3)$ & & \\
\hline College or above & $16(12.5)$ & $5(11.6)$ & $6(14.6)$ & $5(11.4)$ & & \\
\hline Monthly income RMB & & & & & $0.434^{b}$ & 0.980 \\
\hline$\leq ¥ 999$ & $22(17.2)$ & $7(16.3)$ & $8(19.5)$ & $7(15.9)$ & & \\
\hline$¥ 1000 \sim 2000$ & $62(48.4)$ & $20(46.5)$ & $20(48.8)$ & $22(50.0)$ & & \\
\hline$\geq ¥ 2000$ & $44(34.4)$ & $16(37.2)$ & $13(31.7)$ & $15(34.1)$ & & \\
\hline BMl, Mean (SD) & $23.96 \pm 2.03$ & $24.12 \pm 2.13$ & $23.94 \pm 2.02$ & $23.83 \pm 1.96$ & $0.222^{a}$ & 0.801 \\
\hline Symptom duration, Mean (SD) & $8.02 \pm 3.73$ & $8.16 \pm 4.01$ & $7.80 \pm 43.47$ & $8.07 \pm 3.75$ & $0.102^{a}$ & 0.903 \\
\hline Number of affected knees & & & & & $0.798^{b}$ & 0.671 \\
\hline One & $87(68.0)$ & $27(62.8)$ & $29(70.7)$ & $31(70.5)$ & & \\
\hline Two & $41(32.0)$ & $16(37.2)$ & $12(29.3)$ & $13(29.5)$ & & \\
\hline
\end{tabular}


Table 2 Comparisons the average score of pain and physical function among the three groups in different time points $(n=128)$

\begin{tabular}{|c|c|c|c|c|c|c|c|c|c|c|c|}
\hline \multirow{3}{*}{$\begin{array}{l}\text { Primary } \\
\text { outcomes }\end{array}$} & \multirow{3}{*}{$\begin{array}{l}\text { BL } \\
\text { (Mean } \pm S D)\end{array}$} & \multirow{3}{*}{$\begin{array}{l}\text { 3rd MFU } \\
(\text { Mean } \pm \text { SD) }\end{array}$} & \multirow{3}{*}{$\begin{array}{l}\text { 6th MFU } \\
\text { (Mean } \pm \text { SD) }\end{array}$} & \multicolumn{6}{|c|}{ Between groups (QSE vs. BDJ vs. CE) } & \multicolumn{2}{|c|}{ Time $\times$ Group } \\
\hline & & & & \multicolumn{2}{|c|}{$\mathrm{BL}$} & \multicolumn{2}{|c|}{ 3rd MFU } & \multicolumn{2}{|c|}{ 6th MFU } & \multirow[b]{2}{*}{$F$} & \multirow[b]{2}{*}{$P$-valuet } \\
\hline & & & & $F$ & $P$-value & $F$ & $P$-value & $F$ & $P$-value & & \\
\hline WOMAC-pain & & & & 0.045 & 0.956 & 2.875 & 0.060 & 9.071 & $<0.001$ & 28.888 & $<0.001$ \\
\hline QSE $(n=43)$ & $6.91 \pm 2.42$ & $5.47 \pm 2.20$ & $5.53 \pm 2.49$ & & & & & & & & \\
\hline $\operatorname{BDJ}(n=41)$ & $6.76 \pm 2.42$ & $5.71 \pm 2.10$ & $5.46 \pm 2.25$ & & & & & & & & \\
\hline $\mathrm{CE}(n=44)$ & $6.86 \pm 2.24$ & $4.66 \pm 2.08$ & $3.68 \pm 2.13$ & & & & & & & & \\
\hline \multicolumn{2}{|c|}{ WOMAC-physical function } & & & -0.061 & 0.941 & 2.746 & 0.068 & 5.440 & 0.005 & 26.646 & $<0.001$ \\
\hline QSE $(n=43)$ & $18.91 \pm 5.35$ & $16.74 \pm 5.07$ & $16.88 \pm 6.49$ & & & & & & & & \\
\hline $\operatorname{BDJ}(n=41)$ & $18.76 \pm 5.06$ & $16.32 \pm 5.05$ & $16.00 \pm 6.45$ & & & & & & & & \\
\hline $\mathrm{CE}(n=44)$ & $19.14 \pm 4.77$ & $14.41 \pm 4.72$ & $12.59 \pm 6.27$ & & & & & & & & \\
\hline
\end{tabular}

t The repeated measures ANOVA was used to test the time by group interaction effects across the 3 time points of the study

WOMAC Western Ontario and McMaster Universities osteoarthritis index, pain (0-20), physical function (0-68), QSE Quadriceps strengthening exercise, BDJ

Baduanjin qigong, CE The combined exercise of quadriceps strengthening exercise and Baduanjin qigong, BL Baseline, MFU Month of follow-up, SD Standard

deviation; $p<0.05$ was considered statistically significant

of follow-up were significantly reduced when compared to baseline, with the CE group having significantly larger reductions in both measures when compared to the other two groups.

Post-hoc multiple comparison of differences in pain and physical function during the study is shown in Table 3. For the CE group, there were statistically significant reductions in pain intensity and physical function over different time intervals: Baseline vs month 3 of follow-up: pain: 2.205 (CI: 1.896-2.514), physical function: 4.727 (CI: 4.194-5.260). Baseline vs month 6 of follow-up: pain: 3.182 (CI: 2.803-3.561), physical function: 6.545 (CI: 5.598-7.493). Month 3 of follow-up vs month 6 of follow-up: pain: 0.977 (CI: 0.639-1.315), physical function: 1.818 (CI: 0.943-2.694). In the other two groups, statistically significant reductions in both measurements were seen for the same time intervals, except at month 3 of follow-up vs month 6 of follow-up (QSE group: pain: -0.070 (CI: - 0.412-0.272), physical function: -0.140 (CI: $-1.025-0.746)$, BDJ group: pain: -0.244 (CI: $-0.106-0.594)$, physical function: -0.317 (CI: $-0.394-1.028)$ ).

\section{Effects on self-efficacy and HRQoL}

Table 4 shows results for self-efficacy and HRQoL. There were no statistically significant differences among the three groups for any outcome at baseline. After 3 months of follow-up, statistically significant differences

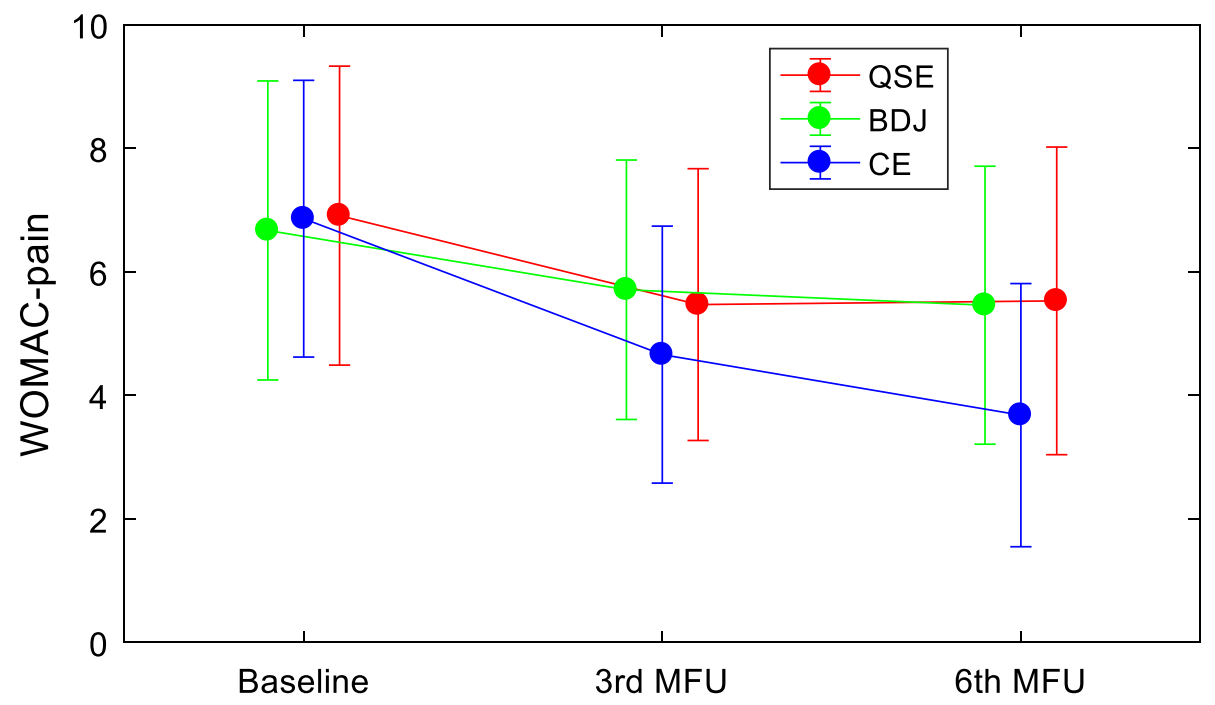

Fig. 2 Trends of pain intensity for the three groups participants at the 3 time points of the study. WOMAC: Western Ontario and McMaster Universities osteoarthritis index; QSE: quadriceps strengthening exercise; BDJ: Baduanjin qigong; CE: the combined exercise of quadriceps strengthening exercise and Baduanjin qigong; MFU: month of follow-up 


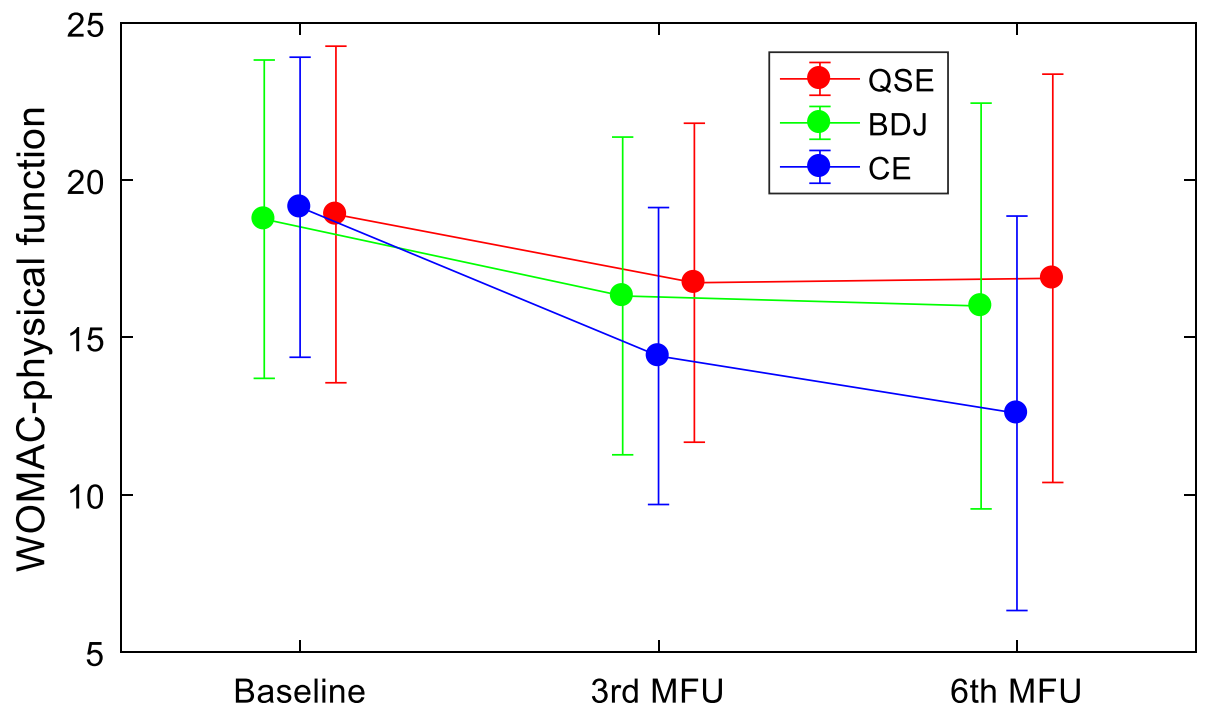

Fig. 3 Trends of physical function for the three groups participants at the 3 time points of the study. WOMAC: Western Ontario and McMaster Universities osteoarthritis index; QSE: quadriceps strengthening exercise; BDJ: Baduanjin qigong; CE: the combined exercise of quadriceps strengthening exercise and Baduanjin qigong; MFU: month of follow-up

were found for self-efficacy $(\mathrm{F}=4.123, P=0.018)$. At 6 months of follow-up, there were statistically significant differences among the three groups in self-efficacy $(\mathrm{F}=12.146, P<0.001)$ and physical component summary $(\mathrm{F}=4.233, P=0.017)$ and mental component summary $(\mathrm{F}=3.890, P=0.023)$ of $\mathrm{SF}-12$.

The analyses of time-by-group-interaction effects on selfefficacy and HRQoL for the length of the whole study are shown in Table 4. The analysis indicated statistically significant time-by-group-interaction effects on self-efficacy $(\mathrm{F}=22.359$, $P<0.001)$, physical component summary $(\mathrm{F}=7.470, P<0.001)$, and mental component summary $(\mathrm{F}=10.207, P<0.001)$.

\section{Discussion}

Since OA cannot be cured [1], lifestyle change, particularly exercise and activity, is vital when managing knee
OA $[10,11]$. Therefore, it is necessary to develop effective exercise intervention programs that facilitate compliance by those with KOA.

Our study suggests that compared to an intervention only involving QSE or BDJ, a combining program of QSE and Baduanjin was more effective in reducing pain intensity in older KOA patients, improving their physical functioning, self-efficacy, and HRQoL. Crucially, the combination exercise program resulted in a better compliance (Figs. 2 and 3).

Research has shown that diminished lower limb muscle strength in KOA patients is associated with disease progression, pain, physical dysfunction, and reduced quality of life $[8,31]$. Thus, quadriceps strengthening can be considered vital in managing KOA [23]. QSE as a

Table 3 SIDAK multiple pairwise comparisons of average score of pain and physical function for the three groups at different time points of the study

\begin{tabular}{|c|c|c|c|c|c|c|}
\hline \multirow{2}{*}{$\begin{array}{l}\text { Primary } \\
\text { outcomes }\end{array}$} & \multicolumn{2}{|l|}{ BL vs. 3rd MFU } & \multicolumn{2}{|l|}{ BL vs. 6th MFU } & \multicolumn{2}{|l|}{ 3rd MFU vs. 6th MFU } \\
\hline & Mean difference $(95 \% \mathrm{Cl})$ & $P$-value & Mean difference $(95 \% \mathrm{Cl})$ & $P$-value & Mean difference $(95 \% \mathrm{Cl})$ & $P$-value \\
\hline \multicolumn{7}{|l|}{ WOMAC-pain } \\
\hline QSE $(n=43)$ & $1.442($ from 1.129 to 1.754 ) & $<0.001$ & 1.372(from 0.988 to 1.756 ) & $<0.001$ & -0.070 (from -0.412 to 0.272 ) & 0.946 \\
\hline $\operatorname{BDJ}(n=41)$ & 1.049(from 0.729 to 1.369 ) & $<0.001$ & $1.293($ from 0.900 to 1.686 ) & $<0.001$ & 0.244 (from -0.106 to 0.594 ) & 0.257 \\
\hline $\mathrm{CE}(n=44)$ & 2.205(from 1.896 to 2.514 ) & $<0.001$ & $3.182($ from 2.803 to 3.561 ) & $<0.001$ & 0.977 (from 0.639 to 1.315 ) & $<0.001$ \\
\hline \multicolumn{7}{|c|}{ WOMAC-physical function } \\
\hline $\mathrm{QSE}(n=43)$ & 2.163(from 1.624 to 2.702) & $<0.001$ & 2.023(from 1.065 to 2.982) & $<0.001$ & -0.140 (from -1.025 to 0.746$)$ & 0.974 \\
\hline $\operatorname{BDJ}(n=41)$ & 2.439 (from 1.887 to 2.991 ) & $<0.001$ & 2.756(from 1.907 to 3.605 ) & $<0.001$ & 0.317 (from -0.394 to 1.028 ) & 0.373 \\
\hline $\mathrm{CE}(n=44)$ & 4.727 (from 4.194 to 5.260 ) & $<0.001$ & 6.545 (from 5.598 to 7.493 ) & $<0.001$ & 1.818 (from 0.943 to 2.694 ) & $<0.001$ \\
\hline
\end{tabular}

WOMAC Western Ontario and McMaster Universities osteoarthritis index, pain (0-20), physical function (0-68), QSE Quadriceps strengthening exercise, BDJ Baduanjin qigong, CE The combined exercise of quadriceps strengthening exercise and Baduanjin qigong, BL Baseline, MFU Month of follow-up, SD Standard deviation, $\mathrm{Cl}$ Confidence interval; $p<0.05$ was considered statistically significant after adjustment 
Table 4 Comparisons of self-efficacy and HRQoL among the three groups in different time points $(n=128)$

\begin{tabular}{|c|c|c|c|c|c|c|c|c|c|c|c|}
\hline \multirow{3}{*}{$\begin{array}{l}\text { Secondary } \\
\text { Outcomes }\end{array}$} & \multirow{3}{*}{$\begin{array}{l}\text { BL } \\
(\text { Mean } \pm \text { SD) }\end{array}$} & \multirow{3}{*}{$\begin{array}{l}\text { 3rd MFU } \\
(\text { Mean } \pm \text { SD) }\end{array}$} & \multirow{3}{*}{$\begin{array}{l}\text { 6th MFU } \\
(\text { Mean } \pm \text { SD) }\end{array}$} & \multicolumn{6}{|c|}{ Between groups (QSE vs. BDJ vs. CE) } & \multicolumn{2}{|c|}{ Time $\times$ Group } \\
\hline & & & & \multicolumn{2}{|c|}{$\mathrm{BL}$} & \multicolumn{2}{|c|}{ 3rd MFU } & \multicolumn{2}{|c|}{ 6th MFU } & \multirow[b]{2}{*}{$F$} & \multirow[b]{2}{*}{$P$-value } \\
\hline & & & & $F$ & $P$-value & $F$ & $P$-value & $F$ & $P$-value & & \\
\hline SF-12 PCS & & & & 0.062 & 0.940 & 1.741 & 0.180 & 4.233 & 0.017 & 7.470 & $<0.001$ \\
\hline QSE $(n=43)$ & $41.35 \pm 9.17$ & $45.21 \pm 9.23$ & $44.33 \pm 10.03$ & & & & & & & & \\
\hline $\mathrm{BDJ}(n=41)$ & $41.62 \pm 8.40$ & $46.88 \pm 10.44$ & $45.88 \pm 11.91$ & & & & & & & & \\
\hline $\mathrm{CE}(n=44)$ & $42.05 \pm 10.21$ & $49.43 \pm 12.04$ & $51.42 \pm 13.58$ & & & & & & & & \\
\hline SF-12 MCS & & & & 0.033 & 0.968 & 2.267 & 0.108 & 3.890 & 0.023 & 10.207 & $<0.001$ \\
\hline $\mathrm{QSE}(n=43)$ & $44.77 \pm 11.81$ & $48.33 \pm 11.38$ & $47.45 \pm 13.97$ & & & & & & & & \\
\hline $\mathrm{BDJ}(n=41)$ & $45.35 \pm 11.84$ & $48.93 \pm 11.28$ & $48.40 \pm 13.68$ & & & & & & & & \\
\hline $\mathrm{CE}(n=4))$ & $45.31 \pm 11.68$ & $52.98 \pm 10.59$ & $54.61 \pm 11.25$ & & & & & & & & \\
\hline ASES-8 & & & & 0.072 & 0.931 & 4.123 & 0.018 & 12.146 & $<0.001$ & 22.359 & $<0.001$ \\
\hline $\operatorname{QSE}(n=43)$ & $4.74 \pm 0.83$ & $5.44 \pm 0.84$ & $5.26 \pm 1.17$ & & & & & & & & \\
\hline $\operatorname{BDJ}(n=41)$ & $4.79 \pm 0.69$ & $5.53 \pm 0.81$ & $5.72 \pm 1.16$ & & & & & & & & \\
\hline $\mathrm{CE}(n=44)$ & $4.80 \pm 0.76$ & $5.92 \pm 0.85$ & $6.48 \pm 1.22$ & & & & & & & & \\
\hline
\end{tabular}

† The repeated measures ANOVA was used to test the time by group interaction effects across the 3 time points of the study

ASES-8 Short form-8 item arthritis self-efficacy scale, SF-12 Short form-12 item health survey questionnaire, SF-12 PCS Physical component summary, SF-12 MCS Mental component summary, QSE Quadriceps strengthening exercise, $B D J$ Baduanjin qigong, $C E$ The combined exercise of quadriceps strengthening exercise and baduanjin qigong, BL Baseline, MFU Month of follow-up, SD Standard deviation; $p<0.05$ was considered statistically significant

widely-used muscle training method $[7,10,11]$. Its beneficial effects on pain relief and functional improvement in KOA patients has been demonstrated by our study (Table 2). The benefits of Baduanjin for joint pain and quality-of-life in KOA patients is similar to those of practicing QSE (Table 3). This is largely consistent with the conclusions of previous studies [32]. Pain and physical function, as measured using WOMAC, improved significantly during follow-up in the $\mathrm{CE}$ group when compared to the other two groups. The greater benefit of combining QSE with BDJ, in addition to the effects of QSE on leg muscle strength, may relate to how BDJ improves function, allowing greater participation in activities, in elderly KOA patients, and how this alters their pain perception.

As a low-impact aerobic exercise with soft movements, Baduanjin stretches and relaxes the musculoskeleton throughout the entire body [16]. Previous studies have suggested that Baduanjin improves fatigue, balance, and physical flexibility in older people [14, 16]. Importantly, partial Baduanjin poses [14, 33, 34] could specifically enhance lower limb muscle strength. For example, "drawing the bow on both sides" contracts hamstring muscles while stretching the quadriceps; "swaying the head and shaking the tail" exercises lower limb adductor muscles; "bouncing on the toes" simultaneously strengthens lower limb anterior and posterior muscles by putting knees together and lifting heels.

Due to the positive effects of Baduanjin movements on lower limb muscle strength, pain and physical functionality improved significantly after training in the $\mathrm{CE}$ group (as measured by WOMAC) (Tables 2, 3).
Maintaining functionality is a main goal of OA treatment $[9,35]$. Good physical function benefits patients allowing them to undertake daily activities and independent social interaction [7]. Quality of life in these patients improved correspondingly [35]. Our findings support combining QSE and Baduanjin interventions in KOA.

As a mind-body exercise, Baduanjin postures can strengthen muscles [16], regulate breathing, inducing a relaxed state of mind [18]. Thus it may help individuals decrease pain sensitivity and alter their perception of pain. Evidence demonstrates that Baduanjin training increases excitation in the middle cerebral cortex, downregulating anxiety and negative emotions [36], and thus promotes mental health [37]. With a peaceful state of mind, individuals are more likely to make correct decisions in self-management [38], and the corresponding impact of pain may likewise be reduced [39]. Such influences help break the "pain cycle" [15], enhancing patient confidence when coping with disease or discomfort [13].

Our findings suggest that a QSE plus Baduanjin intervention has more a positive effect on self-efficacy and the QoL of KOA patients than does QSE or BDJ alone (Table 4). This is to be expected, as previous studies have also indicated positive associations between selfefficacy and pain in those suffering with chronic musculoskeletal pain [40]. Veenhof (2006) identifies that to ensure good compliance in OA patients, the ultimate goal of exercise intervention is to integrate the exercises into daily life [41]. However, the optimal way to achieve this remains an open question. The reality is that this goal is seldom achieved by asking patients to perform 
monotonous exercises repetitively [12]. Instead, it is essential to mobilize multifaceted support around the patient, such as encouragement from peers [42-44].

In our study, participants were required to practice Baduanjin using a group-based form, so that they could share successful personal experience in OA management [44] and to provide mutual encouragement and support [42]; participants in the QSE group practiced QSE at home alone. Moreover, group exercise may make patients feel exercise is not a treatment but a part of daily life, eliminating their awareness of a patients' role and enhancing their sense initiative in KOA management. Thus, subjects in the CE group had higher levels of selfefficacy and exhibited better exercise adherence.

A review indicated that choosing exercises based on patient preference can increase adherence to exercise [12]. Baduanjin is very popular among older adults, but most such patients with KOA were concerned that practicing Baduanjin might aggravate joint symptoms [45]. In our study, such concerns were deprecated through appropriate guidance by researchers. Our study indicates that practicing Baduanjin was safe and effective for older patients with KOA, and that participants in the QSE plus Baduanjin group demonstrated greater adherence. Adherence to exercise was a key predictor of beneficial long-term outcome from exercise [8]. When designing the study, our concern was that the greater amount of exercise expected of the intervention group might reduce compliance. However, at 6 months of follow-up, results indicated that the $\mathrm{CE}$ group exhibited greater enthusiasm, participation, and adherence.

There were several limitations in our study. First, participants were only recruited from urban communities, while rural residents were not included. Ideally, future investigation should include rural residents from diverse settings, so that a more detailed and conclusive assessment of the effectiveness of the program can be obtained. Secondly, patients who agreed to participate in the study might be more concerned about their health, resulting in higher completion and lower patient attrition rates, and ultimately achieving better intervention results. Thirdly, to maintain safety, we only recruited older patients with mild to moderate pain on NRS. Future study should include patients with severe pain to confirm the efficacy of QSE plus Baduanjin treatment in reducing pain and improving other health outcomes among such patients. Finally, a longer period of followup should be used to test long-term compliance and outcomes. Larger, more strictly designed trials are needed to confirm our findings.

\section{Conclusions}

To conclude, exercise programs combining strengthening exercise and Baduanjin promote positive lifestyle changes with increased physical activity. Results indicate that patients with KOA in the QSE plus Baduanjin group exhibited significantly greater pain reduction, greater improvement in physical function and self-efficacy, and a better physical and mental health status than those in the QSE or BDJ only group. Likewise, outcome improvements in the QSE plus Baduanjin group are better sustained. When practiced correctly, Baduanjin exercise also appears to be safe and effective for patients with KOA. This study provides evidence of the value of exercise programs for patients with KOA. Due to the small sample size of our study, our conclusion should be treated with caution, indicating the need for longer, larger, and more sophisticated studies to evaluate the efficacy of combining Baduanjin exercise with QSE.

\section{Abbreviations}

OA: Osteoarthritis; KOA: Knee osteoarthritis; NRS: Numeric rating scale;

BMI: Body mass index; WOMAC: Western Ontario and McMaster Universities Osteoarthritis Index; ASES-8: Short Form-8 item Arthritis Self-Efficacy Scale; SF-12: Short Form-12 item health survey questionnaire; SF-12 PCS: Physical component summary of SF-12; SF-12 MCS: Mental component summary of SF-12; QSE: Quadriceps strengthening exercises; BDJ: Baduanjin qigong.; CE: The combined exercise of quadriceps strengthening exercise and baduanjin qigong; HRQoL: Health related quality of life; BL: Baseline; MFU: Month of follow-up; Cl: Confidence interval; SD: Standard deviation

\section{Supplementary Information}

The online version contains supplementary material available at https://doi. org/10.1186/s12891-021-04179-8.

Additional file 1. The guidelines for practicing quadriceps strengthening exercises and Baduanjin qigong for $\mathrm{KOA}$ patients in this study.

Additional file 2. The dataset supporting the conclusions of this article. Additional file 3. CONSORT 2010 checklist of information to include when reporting a randomized trial.

\section{Acknowledgements}

The authors would like to thank the participating patients and health professionals involved in this study. We would like to thank Xinguang Yang for helping organize and coordinate community interventions; Dr. Hongjuan Han for her assistance with the statistical analysis; and Dr. Liang Chen for editing our figures.

\section{Authors' contributions}

WF participated in the design of the study, performed the data analysis, and drafted the manuscript. ZX was responsible for the resources and project administration. TX performed the statistical analyses and validated the study. XF supervised the study. ZM collected and analyzed patient data. YK and JN performed the assessment and exercise interventions for patients. DZ was a major contributor in the design of this study and revised the manuscript. All authors contributed to and approved the final manuscript.

\section{Funding}

This work was approved as a self-funded project of the Hebei Educational Committee (reference number: ZC2016042). We affirm that we have no financial affiliation (including research funding) or involvement with any commercial organization that has a direct financial interest in any matter included in this manuscript.

Availability of data and materials

The dataset supporting the conclusions of this article can be accessed through Additional file 2. 


\section{Declarations}

\section{Ethics approval and consent to participate}

The study protocol was approved by the Ethics Committee of the North China University of Science and Technology (reference number: 2019073). All participants provided informed, written consent.

\section{Consent for publication}

The model for the Baduanjin exercise photos gave her consent to publish these photos in the present study protocol. Her written consent is available upon request.

\section{Competing interests}

The authors declare that they have no competing interests.

\section{Author details}

'School of Nursing, Shanxi Medical University, 56 Xinjian Road, Yingze District, Taiyuan 030001, China. ${ }^{2}$ College of Nursing and Rehabilitation, North China University of Science and Technology, 21 Bohai Road, Caofeidian District, Tangshan 063210, China. ${ }^{3}$ Department of Joint Surgery, The Second Hospital of Tangshan, 21 Jianshe Road, Lubei District, Tangshan 063000, China.

\section{Received: 30 August 2020 Accepted: 16 March 2021}

\section{Published online: 29 March 2021}

\section{References}

1. Hawker GA. Osteoarthritis is a serious disease. Clin Exp Rheumatol. 2019; 120(5):3-6.

2. Cross M, Smith E, Hoy D, Nolte S, Ackerman I, Fransen M, et al. The global burden of hip and knee osteoarthritis: estimates from the global burden of disease 2010 study. Ann Rheum Dis. 2014;73(7):1323-30. https://doi.org/1 0.1136/annrheumdis-2013-204763.

3. Vos T, Flaxman AD, Naghavi M, Lozano R, Michaud C, Ezzati M, et al. Years lived with disability (YLDs) for 1160 sequelae of 289 diseases and injuries 1990-2010: a systematic analysis for the global burden of disease study 2010. Lancet. 2012;380(9859):2163-96. https://doi.org/10.1016/S0140-6736(12 )61729-2.

4. Sridhar MS, Jarrett CD, Xerogeanes JW, Labib SA. Obesity and symptomatic osteoarthritis of the knee. J Bone Joint Surg Br. 2012;94(4):433-40.

5. Liu Q, Wang S, Lin J, Zhang Y. The burden for knee osteoarthritis among Chinese elderly: estimates from a nationally representative study. Osteoarthr Cartil. 2018;26(12):1636-42. https://doi.org/10.1016/j.joca.2018.07.019.

6. Brown A. FNP-BC. Holistic/integrative interventions relieve knee osteoarthritis pain in older adults. J Nurse Pract. 2019;16(1):57-9.

7. Chen H, Zheng X, Huang H, Liu C, Wan Q, Shang S. The effects of a homebased exercise intervention on elderly patients with knee osteoarthritis: a quasi-experimental study. BMC Musculoskelet Disord. 2019;20(1):160. https:// doi.org/10.1186/s12891-019-2521-4.

8. Roddy E, Zhang W, Doherty M, Arden NK, Barlow J, Birrell F, et al. Evidencebased recommendations for the role of exercise in the management of osteoarthritis of the hip or knee-the MOVE consensus. Rheumatology (Oxford). 2005;44(1):67-73. https://doi.org/10.1093/rheumatology/keh399.

9. Hochberg MC, Altman RD, April KT, Benkhalti M, Guyatt G, MCGowan J, et al. American College of Rheumatology 2012 recommendations for the use of nonpharmacologic and pharmacologic therapies in osteoarthritis of the hand, hip, and knee. Arthritis Care Res (Hoboken). 2012;64(4):465-74. https:// doi.org/10.1002/acr.21596.

10. McKnight PE, Kasle S, Going S, Villanueva I, Cornett M, Farr J, et al. A comparison of strength training, self-management, and the combination for early osteoarthritis of the knee. Arthritis Care Res. 2010;62(1):45-53. https:// doi.org/10.1002/acr.20013.

11. Baker K, LaValley MP, Brown C, Felson DT, Ledingham A, Keysor JJ. Efficacy of computer-based telephone counseling on long-term adherence to strength training in elders with knee osteoarthritis: a randomized trial. Arthritis Care Res. 2019:72(7):982-90. https://doi.org/10.1002/acr.23921.

12. Aitken $D$, Buchbinder $R$, Jones $G$, Winzenberg $T$. Interventions to improve adherence to exercise for chronic musculoskeletal pain in adults. Aust Fam Physician. 2015;44(1-2):39-42.

13. Goh SL, Persson MSM, Stocks J, Hou Y, Welton NJ, Lin J, et al. Relative efficacy of different exercises for pain, function, performance and quality of life in knee and hip osteoarthritis: systematic review and network metaanalysis. Sports Med. 2019;49(5):743-61. https://doi.org/10.1007/s40279-01901082-0.

14. Zeng ZP, Liu YB, Fang J, Liu Y, Luo J, Yang M. Effects of Baduanjin exercise for knee osteoarthritis: a systematic review and meta-analysis. Complement Ther Med. 2020:48:102279. https://doi.org/10.1016/j.ctim.2019.102279.

15. Xie Y, Guo F, Lu Y, Guo Y, Wei G, Lu L, et al. A 12-week Baduanjin qigong exercise improves symptoms of ankylosing spondylitis: a randomized controlled trial. Complement Ther Clin Pract. 2019;36:113-9. https://doi. org/10.1016/j.ctcp.2018.12.007.

16. Zou L, Yeung A, Quan X, Boyden SD, Wang H. A systematic review and meta-analysis of mindfulness-based (Baduanjin) exercise for alleviating musculoskeletal pain and improving sleep quality in people with chronic diseases. Int J Environ Res Public Health. 2018;15(2):206. https://doi.org/10.33 90/ijerph15020206.

17. Li H, Ge D, Liu S, Zhang W, Wang JG, Si JH, et al. Baduanjin exercise for low back pain: a systematic review and meta-analysis. Complement Ther Med. 2019;43:109-16. https://doi.org/10.1016/j.ctim.2019.01.021.

18. Cheng FK. Effects of Baduanjin on mental health: a comprehensive review. J Bodyw Mov Ther. 2015;19(1):138-49. https://doi.org/10.1016/j.jbmt.2014.11. 001.

19. Kan LD, Zhang JQ, Yang YH, Wang P. The Effects of Yoga on Pain, Mobility, and Quality of Life in Patients with Knee Osteoarthritis: A Systematic Review. Evid Based Complementray Altern Med. 2016;(2016-9-29):1-10. https://doi. org/10.1155/2016/6016532.

20. Brosseau L, Taki J, Desjardins B, Thevenot O, Fransen M, Wells GA, et al. The Ottawa panel clinical practice guidelines for the management of knee osteoarthritis. Part three: aerobic exercise programs. Clin Rehabil. 2017;31(5): 612-24. https://doi.org/10.1177/0269215517691085.

21. Aglamiş B, Toraman NF, Yaman $\mathrm{H}$. Change of quality of life due to exercise training in knee osteoarthritis: SF-36 and WOMAC. J Back Musculoskelet Rehabil. 2009;22(1):43-5, 47-8, 46-8. https://doi.org/10.3233/BMR-2009-0219.

22. Egwu O, Ayanniyi O, Adegoke B, Olagbegi O, Ogwumike O, Odole A. Effect of self-management education versus quadriceps strengthening exercises on pain and function in patients with knee osteoarthritis. Human Movement. 2018;19(3):64-74. https://doi.org/10.5114/hm.2018.76081.

23. Imoto AM, Peccin MS, Trevisani VF. Quadriceps strengthening exercises are effective in improving pain, function and quality of life in patients with osteoarthritis of the knee. Acta Ortop Bras. 2012;20(3):174-9. https://doi. org/10.1590/S1413-78522012000300008.

24. Bellamy N, Buchanan WW, Goldsmith CH, Campbell J, Stitt LW. Validation of WOMAC: a health status instrument for measuring clinically important patient relevant outcomes to antirheumatic drug therapy in patients with osteoarthritis of the hip or knee. J Rheumatol. 1988;15(12):1833-40.

25. Xie F, Li SC, Goeree R, Tarride JE, Reilly DO, Lo NN, et al. Validation of Chinese Western Ontario and McMaster universities osteoarthritis index (WOMAC) in patients scheduled for total knee replacement. Qual Life Res. 2008;17(4):595-601. https://doi.org/10.1007/s11136-008-9340-7.

26. Lorig K, Chastain RL, Ung E, Shoor S, Holman HR. Development and evaluation of a scale to measure perceived self-efficacy in people with arthritis. Arthritis Rheum. 1989;32(1):37-44. https://doi.org/10.1002/anr.1 780320107.

27. Gao L, Zhang XC, Li MM, Yuan JQ, Cui XJ, Shi BX. Psychometric properties of the Chinese version of arthritis self-efficacy scale-8 (ASES-8) in a rheumatoid arthritis population. Rheumatol Int. 2017;37(5):751-6. https://doi.org/10.1 007/s00296-016-3640-y.

28. Ware JJ, Kosinski M, Keller SD. A 12-item short-form health survey: construction of scales and preliminary tests of reliability and validity. Med Care. 1996;34(3):220-33. https://doi.org/10.1097/00005650-199603000-00003.

29. Jiang $W H$, Zhang YN, Yan FH, Liu H, Gao R. Effectiveness of a nurse-led multidisciplinary self-management program for patients with coronary heart disease in communities: a randomized controlled trial. Patient Educ Couns. 2019;103(4):854-63. https://doi.org/10.1016/j.pec.2019.11.001.

30. Webster KE, Feller JA. Comparison of the short form-12 (SF-12) health status questionnaire with the SF-36 in patients with knee osteoarthritis who have replacement surgery. Knee Surg Sports Traumatol Arthrosc. 2016;24(8):26206. https://doi.org/10.1007/s00167-015-3904-1.

31. Brosseau L, Taki J, Desjardins B, Thevenot O, Fransen M, Wells GA, et al. The Ottawa panel clinical practice guidelines for the management of knee osteoarthritis. Part two: strengthening exercise programs. Clin Rehabil. 2017; 31(5):596-611. https://doi.org/10.1177/0269215517691084. 
32. Chen FN, Wu WM, Le YM. Application of Baduanjin in functional exercise of elderly patients with chronic knee osteoarthritis and its effect on exercise capacity and self-management effectiveness [J]. Chin J Health Manage. 2020;14(06):556-9.

33. Qin KH, Guo YY, Yang SQ, Ye QB. Analysis on mechanism of Baduanjin in improving knee osteoarthritis in middle-aged and old people. J Chengdu Univ TCM. 2019;42(03):9-11.

34. Hu XL, Guan XF, Yang SZ. Analysis of knee osteoarthritis adjuvant treated by Baduanjin. J Liaoning Univ TCM. 2019;21(2):80-2.

35. Cheung C, Wyman JF, Bronas U, McCarthy T, Rudser K, Mathiason MA. Managing knee osteoarthritis with yoga or aerobic/strengthening exercise programs in older adults: a pilot randomized controlled trial. Rheumatol Int. 2017;37(3):389-98. https://doi.org/10.1007/s00296-016-3620-2.

36. Jing $L$, Jin $Y$, Zhang $X$, Wang $F$, Song $Y$, Xing $F$. The effect of Baduanjin qigong combined with CBT on physical fitness and psychological health of elderly housebound. Medicine (Baltimore). 2018;97(51):e13654. https://doi. org/10.1097/MD.0000000000013654

37. Zou L, Yeung A, Quan X, Hui SS, Hu X, Chan JSM, et al. Mindfulness-based Baduanjin exercise for depression and anxiety in people with physical or mental illnesses: a systematic review and meta-analysis. Int J Environ Res Public Health. 2018;15(2):321. https://doi.org/10.3390/ijerph15020321.

38. Brosseau L, Taki J, Desjardins B, Thevenot O, Fransen M, Wells GA, et al. The Ottawa panel clinical practice guidelines for the management of knee osteoarthritis. Part one: introduction, and mind-body exercise programs. Clin Rehabil. 2017;31(5):582-95. https://doi.org/10.1177/0269215517691083.

39. Morone NE, Greco CM. Mind-body interventions for chronic pain in older adults: a structured review. Pain Med. 2007;8(4):359-75. https://doi.org/1 0.1111/j.1526-4637.2007.00312.x.

40. Martinezcalderon J, Zamoracampos C, Navarroledesma S, Luquesuarez A. The role of self-efficacy on the prognosis of chronic musculoskeletal pain: a systematic review. J Pain. 2018;19(1):10-34. https://doi.org/10.1016/j.jpain.2 017.08.008.

41. Veenhof C, Köke AJ, Dekker J, Oostendorp RA. Effectiveness of behavioral graded activity in patients with osteoarthritis of the hip and/or knee: a randomized clinical trial. Arthritis Rheum. 2006;55(6):925-34. https://doi. org/10.1002/art.22341.

42. Wang FL, Dong SL, Zhang YM, Zhang XL, Xing FM. Intervention effect of peer education to the elderly patients with knee osteoarthritis in community. Modern Prev Med. 2015;42(5):827-30.

43. Debussche X, Besançon S, BalcouDebussche M, Ferdynus C, Delisle H, Huiart $\mathrm{L}$, et al. Structured peer-led diabetes self-management and support in a low-income country: The ST2EP randomised controlled trial in Mali. PLoS One. 2018;13(1):e0191262. Published 2018 Jan 22. https://doi.org/10.1371/ journal.pone.0191262.

44. Best KL, Miller WC, Eng JJ, Routhier F. Systematic review and meta-analysis of peer-led self-management programs for increasing physical activity. Int J Behav Med. 2016;23(5):527-38. https://doi.org/10.1007/s12529-016-9540-4.

45. Gunn AH, Schwartz TA, Arbeeva LS, Callahan LF, Golightly Y, Goode A, et al. Fear of movement and associated factors among adults with symptomatic knee osteoarthritis. Arthritis Care Res (Hoboken). 2017:69(12):1826-33. https://doi.org/10.1002/acr.23226.

\section{Publisher's Note}

Springer Nature remains neutral with regard to jurisdictional claims in published maps and institutional affiliations.

Ready to submit your research? Choose BMC and benefit from:
- fast, convenient online submission
- thorough peer review by experienced researchers in your field
- rapid publication on acceptance
- support for research data, including large and complex data types
- gold Open Access which fosters wider collaboration and increased citations
- maximum visibility for your research: over 100M website views per year
At BMC, research is always in progress.
Learn more biomedcentral.com/submissions

